\title{
PHENOMENON OF THE TIME-REVERSAL-VIOLATING PHOTON POLARIZATION PLANE ROTATION BY A GAS PLACED TO AN ELECTRIC FIELD
}

\author{
V.G.Baryshevsky
}

1. Violation of time reversal symmetry has been observed only in $K_{0}$-decay many years ago [1], and remains one of the great unsolved problems in elementary particle physics. Since the discovery of the CP-violation in decay of $K_{0}$-mesons, a few attempts have been undertaken to observe this phenomenon experimentally in different processes. However, that experiments have not been successful. At the present time novel more precise experimental schemes are actively discussed: observation of the atom [2] and neutron [3] electric dipole moment, T-violating (time reversal) atom (molecule) spin rotation in a laser wave and T-violating optical activity of an atomic or molecular gas [4. 5].

According to [6] the violation of the time reversal symmetry stipulates the appearance of the new optical phenomenon: the photon polarization plane rotation and circular dichroism in an optical homogeneous isotropic matter placed to an electric field. This T-odd phenomenon is the kinematic analog of the famous T-even phenomenon of the photon polarization plane rotation in a matter placed to a magnetic field (Faraday phenomenon).

In the present paper T-odd phenomenon of the photon polarization plane rotation (circular dichroism) is considered for an atomic (molecular) gas placed to an electric field.

The expression for the $\mathrm{T}$ non-invariant polarizability of an atom (molecule) placed to an electric field is obtained. It is shown that the T-odd plane rotation angle $\vartheta_{T}$ increases when the interaction energy of an atom (molecule) with an electric field is the same order as the opposite parity levels spacing.

2. In accordance with [4, 5, 6] the T-reversal violating dielectric permittivity tensor $\varepsilon_{i k}$ for an optical diluted matter $\left(\varepsilon_{i k}-\delta_{i k} \ll 1, \delta_{i k}\right.$ is the Kronecker symbol) is given by

$$
\varepsilon_{i k}=\delta_{i k}+\chi_{i k}=\delta_{i k}+\frac{4 \pi \rho}{k^{2}} f_{i k}(0),
$$

where $\chi_{i k}$ is the polarizability tensor of a matter, $\rho$ is the number of atoms (molecules) per $\mathrm{cm}^{3}, k$ is the photon wave number; $f_{i k}(0)$ is the tensor part of the zero angle amplitude of elastic coherent scattering of a photon by an atom (molecule) $f(0)=f_{i k}(0) e_{i}^{\prime *} e_{k}$. Here $\vec{e}$ and $\vec{e}^{\prime}$ are the polarization vectors of initial and scattered photons. Indices $i=1,2,3$ are referred to coordinates $x, y, z$ respectively, repeated indices imply summation.

Let photons are scattered by nonpolarized atoms (molecules) interacted with an electric field $\vec{E}$. 
The amplitude $f_{i k}(0)$ can be written as [6]:

$$
f_{i k}(0)=f_{i k}^{e v}+\frac{\omega^{2}}{c^{2}}\left[i \beta_{s}^{P} \varepsilon_{i k l} n_{\gamma l}+i \beta_{E}^{T} \varepsilon_{i k l} n_{E l}\right],
$$

where $f_{i k}^{e v}$ is the T, $\mathrm{P}$ even (invariant) part of $f_{i k}(0), \beta_{s}^{P}$ is the scalar Pviolating polarizability of an atom (molecule), $\beta_{E}^{T}$ is the scalar $\mathrm{T}$ and $\mathrm{P}$ violating polarizability of an atom (molecule), $\varepsilon_{i k l}$ is the totally antisymmetrical unit tensor of the rank three, $\vec{n}_{\gamma}=\frac{\vec{k}}{k}, \vec{k}$ is the photon wave vector, $\vec{n}_{E}=\frac{\vec{E}}{E}$.

The term proportional to $\beta_{s}^{P}$ describes the T-invariant $\mathrm{P}$-violating light polarization plane rotation (and circular dichroism) in a gas [7].

The corresponding refractive index $N$ can be written in the following form convenient for the further analysis:

$$
N=1+\frac{2 \pi \rho}{k^{2}}\left\{f_{i k}^{e v}(0) e_{i}^{*} e_{k}+i \frac{\omega^{2}}{c^{2}}\left(\beta_{s}^{P} \vec{n}_{\gamma}+\beta_{E}^{T} \vec{n}_{E}\right)\left[\vec{e}^{*} \times \vec{e}\right]\right\} .
$$

The unit vectors describing the circular polarization of photons are: $\vec{e}_{+}=$ $-\frac{\vec{e}_{1}+i \vec{e}_{2}}{\sqrt{2}}$ for the right and, $\vec{e}_{-}=\frac{\vec{e}_{1}-i \vec{e}_{2}}{\sqrt{2}}$ for the left circular polarization, where $\vec{e}_{1} \perp \vec{e}_{2}, \vec{e}_{2}=\left[\vec{n}_{\gamma} \times \vec{e}_{1}\right]$ are the unit polarization vectors of a linearly polarized photon, $\left[\vec{e}_{1} \times \vec{e}_{2}\right]=\vec{n}_{\gamma}, \vec{e}_{1}=-\frac{\vec{e}_{+}-\vec{e}_{-}}{\sqrt{2}}$, $\vec{e}_{2}=-\frac{\vec{e}_{+}+\vec{e}_{-}}{i \sqrt{2}}$.

Let an electromagnetic wave propagates in a gas along the direction of the electric field $\vec{E}$.

The refractive indices for the right $N_{+}$and the left $N_{-}$circular polarized photons can be written as:

$$
N_{ \pm}=1+\frac{2 \pi \rho}{k^{2}} f_{ \pm}(0)=1+\frac{2 \pi \rho}{k^{2}}\left\{f^{e v}(0) \mp \frac{\omega^{2}}{c^{2}}\left[\beta_{s}^{P}+\beta_{E}^{T}\left(\vec{n}_{E} \vec{n}_{\gamma}\right)\right]\right\}
$$

where $f_{+}(0)\left(f_{-}(0)\right)$ is the zero angle amplitude of the elastic coherent scattering of a right (left) circular polarized photon by an atom (molecule).

Let photons with the linear polarization $\vec{e}_{1}=-\frac{\vec{e}+\overrightarrow{e_{-}}}{\sqrt{2}}$ fall in a gas. The polarization vector of a photon in a gas $\vec{e}_{1}^{\prime}$ can be written as:

$$
\begin{aligned}
\vec{e}_{1}^{\prime} & =-\frac{\vec{e}_{+}}{\sqrt{2}} e^{i k N_{+} L}+\frac{\vec{e}_{-}}{\sqrt{2}} e^{i k N_{-} L}= \\
& =e^{\frac{1}{2} i k\left(N_{+}+N_{-}\right) L}\left\{\vec{e}_{1} \cos \frac{1}{2} k\left(N_{+}-N_{-}\right) L-\vec{e}_{2} \sin \frac{1}{2} k\left(N_{+}-N_{-}\right) L\right\},
\end{aligned}
$$

where $L$ is the photon propagation length in a medium. 
As one can see, the photon polarization plane rotates in a gas. The angle of rotation $\vartheta$ is

$$
\begin{aligned}
\vartheta & =\frac{1}{2} k \operatorname{Re}\left(N_{+}-N_{-}\right) L=\frac{\pi \rho}{k} \operatorname{Re}\left[f_{+}(0)-f_{-}(0)\right]= \\
& =-\frac{2 \pi \rho \omega}{c}\left[\beta_{s}^{P}+\beta_{E}^{T}\left(\vec{n}_{E} \vec{n}_{\gamma}\right)\right],
\end{aligned}
$$

where $\operatorname{Re} N_{ \pm}$is the real part of $N_{ \pm}$.

It should be noted that $\vartheta>0$ corresponds to the right and $\vartheta<0$ corresponds to the left rotation of the light polarization plane, where the right (positive) rotation is the rotation recording by the light observer as the clockwise one.

In accordance with (6) the T-odd interaction results in the photon polarization plane rotation around the electric field $\vec{E}$ direction. The angle of rotation is proportional to the polarizability $\beta_{E}^{T}$ and $\vec{E}, \vec{n}_{\gamma}$ correlation. Together with the T-odd effect there is the T-even P-odd polarization plane rotation phenomenon determining by the polarizability $\beta_{s}^{P}$ and independent on the $\vec{E}, \vec{n}_{\gamma}$ correlation. The T-odd rotation dependence on the electric field $\vec{E}$ orientation relatively to the $\vec{n}_{\gamma}$ direction allows to distinguish T-odd and T-even P-odd phenomena experimentally.

The refractive indices $N_{ \pm}$have both real and imaginary parts. The imaginary parts of the refractive indices $\left(\operatorname{Im} N_{ \pm} \sim \operatorname{Im} \beta_{E}^{T}\left(\vec{n}_{E} \vec{n}_{\gamma}\right)\right.$ ) are responsible for the T-reversal violating circular dichroism. Due to the this process linearly polarized photons take circular polarization. The sign of the circular polarization depends on the sign of scalar production $\left(\vec{n}_{E} \vec{n}_{\gamma}\right)$ that allows to separate T-odd circular dichroism from P-odd T-even circular dichroism. The last one is proportional to $\operatorname{Im} \beta_{s}^{P}$.

3. In order to estimate the magnitude of the effect one should obtain the T-odd polarizability $\beta_{E}^{T}$ or (that is actually the same, see $(2,6)$ ) the amplitude $f_{ \pm}(0)$ of elastic coherent scattering of a photon by an atom (molecule).

According to quantum electrodynamics the elastic coherent scattering at the zero angle can be considered as the succession of two processes: the first one is the absorption of the initial photon (momentum $\vec{k}$ ) and the transition of the atom (molecule) from the initial state $\left|N_{0}\right\rangle$ with the energy $E_{N_{0}}$ to an intermediate state $|F\rangle$ with an energy $E_{F}$; the second is the transition of the atom (molecule) from the state $|F\rangle$ to the final state $\left|F^{\prime}\right\rangle=\left|N_{0}\right\rangle$ and radiation of the photon with the momentum $\vec{k}^{\prime}=\vec{k}$.

Let $H_{A}$ is the atom (molecule) Hamiltonian considering the weak interaction of electrons and nucleus and the electromagnetic interaction of an atom (molecule) with the external electric field $\vec{E}$. It determines the system of eigenfunctions $|F\rangle$ and eigenvalues $E_{F}$ :

$$
H_{A}|F\rangle=E_{F}|F\rangle .
$$

The matrix element of the process determining the scattering amplitude in 
forward direction in the electric dipole approximation can be written as [8]:

$$
\mathfrak{M}=\sum_{F}\left\{\frac{\left\langle N_{0}\left|\vec{d} \vec{e}^{*}\right| F\right\rangle\left\langle F|\vec{d} \vec{e}| N_{0}\right\rangle}{E_{F}-E_{N_{0}}-\hbar \omega}+\frac{\left\langle N_{0}|\vec{d} \vec{e}| F\right\rangle\left\langle F|\vec{d} \vec{e} *| N_{0}\right\rangle}{E_{F}-E_{N_{0}}+\hbar \omega}\right\},
$$

where $\vec{d}$ is the electric dipole transition operator, $\omega$ is the photon frequency.

Let us remind, that the atom (molecule) exited energy levels are quasistationary: $E_{F}=E_{F}^{(0)}-\frac{i}{2} \Gamma_{F}, E_{F}^{(0)}$ is the atom (molecule) level energy, $\Gamma_{F}$ is the level width.

The matrix element (8) can be written as:

$$
\mathfrak{M}=\alpha_{i k} e_{i}^{*} e_{k},
$$

where the tensor of dynamical atom (molecule) polarizability $\alpha_{i k}$ has the form

$$
\alpha_{i k}=\sum_{F}\left\{\frac{\left\langle N_{0}\left|d_{i}\right| F\right\rangle\left\langle F\left|d_{k}\right| N_{0}\right\rangle}{E_{F}-E_{N_{0}}-\hbar \omega}+\frac{\left\langle N_{0}\left|d_{k}\right| F\right\rangle\left\langle F\left|d_{i}\right| N_{0}\right\rangle}{E_{F}-E_{N_{0}}+\hbar \omega}\right\}
$$

The tensor $\alpha_{i k}$ can be expanded over the irreducible parts as

$$
\alpha_{i k}=\alpha_{0} \delta_{i k}+\alpha_{i k}^{s}+\alpha_{i k}^{a},
$$

where $\alpha_{0}=\frac{1}{3} \sum_{i} \alpha_{i i}$ is the scalar, $\alpha_{i k}^{s}=\frac{1}{2}\left(\alpha_{i k}+\alpha_{k i}\right)-\alpha_{0} \delta_{i k}$ is the symmetrical tensor of rank two, $\alpha_{i k}^{a}=\frac{1}{2}\left(\alpha_{i k}-\alpha_{k i}\right)$ is the antisymmetrical tensor of rank two.

$$
\alpha_{i k}^{a}=\frac{\omega}{\hbar} \sum_{F}\left\{\frac{\left\langle N_{0}\left|d_{i}\right| F\right\rangle\left\langle F\left|d_{k}\right| N_{0}\right\rangle-\left\langle N_{0}\left|d_{k}\right| F\right\rangle\left\langle F\left|d_{i}\right| N_{0}\right\rangle}{\omega_{F N_{0}}^{2}-\omega^{2}}\right\},
$$

where $\omega_{F N_{0}}=\frac{E_{F}-E_{N_{0}}}{\hbar}$.

Let atoms (molecules) are nonpolarized. The antisymmetrical part of polarizability (12) is equal to zero in the absence of T- and P- odd interactions. Let us remind that for P-odd and T-even interactions the antisymmetrical part of polarizability differs from zero when we consider both the electric and magnetic dipole transitions only [7].

We can calculate the antisymmetrical part $\alpha_{i k}^{a}$ of the tensor of dynamical atom (molecule) polarizability $\alpha_{i k}$, and as a result, to obtain the expression for $\beta_{E}^{T}$ by the following way. According to $(4,6)$ the magnitude of the T-odd effect is determined by the polarizability $\beta_{E}^{T}$ or (that is actually the same, see $(2,6)$ ) by the amplitude $f_{ \pm}(0)$ of elastic coherent scattering of a photon by an atom (molecule). When $\vec{n}_{E} \| \vec{n}_{\gamma}$ the amplitude $f_{ \pm}(0)$ in dipole approximation can be written as $f_{ \pm}=\mp \frac{\omega^{2}}{c^{2}} \beta_{E}^{T}$. As a result, in order to obtain the amplitude $f_{ \pm}$ 
, the matrix element $(8,9)$ for photon polarization states $\vec{e}=\vec{e}_{ \pm}$should be find.

The electric dipole transition operator $\vec{d}$ can be written in the form:

$$
\vec{d}=d_{+} \vec{e}_{+}+d_{-} \vec{e}_{-}+d_{z} \vec{n}_{\gamma},
$$

with $\vec{d}_{+}=-\frac{d_{x}-i d_{y}}{\sqrt{2}}, \vec{d}_{-}=\frac{d_{x}+i d_{y}}{\sqrt{2}}$.

Let $\vec{e}=\vec{e}_{+}$. Using $(8,9)$ we can write for polarizability $\beta_{E}^{T}$ the following expression:

$$
\beta_{E}^{T}=\frac{\omega}{\hbar} \sum_{F}\left\{\frac{\left\langle N_{0}\left|d_{-}\right| F\right\rangle\left\langle F\left|d_{+}\right| N_{0}\right\rangle-\left\langle N_{0}\left|d_{+}\right| F\right\rangle\left\langle F\left|d_{-}\right| N_{0}\right\rangle}{\omega_{F N_{0}}^{2}-\omega^{2}}\right\} .
$$

The more detailed expression for atom (molecule) wave functions is necessary for the further calculations of the polarizability. The weak interactions constant is very small. Therefore, we can use the perturbation theory. Let $|f, E\rangle$ is the wave function of an atom (molecule) interacting with an electric field $\vec{E}$ in the absence of weak interactions $\left(V_{w}=0\right)$. Switch on weak interaction $\left(V_{w} \neq 0\right)$. According to the perturbation theory [8] the wave function $|F\rangle$ in this case can be written as:

$$
|F\rangle=|f, \vec{E}\rangle+\sum_{n} \frac{\left\langle n, \vec{E}\left|V_{w}\right| f, \vec{E}\right\rangle}{E_{f}-E_{n}}|n, \vec{E}\rangle
$$

From $(14,15)$ for the polarizability $\beta_{E}^{T}$ we obtain :

$$
\begin{gathered}
\beta_{E}^{T}=\frac{\omega}{\hbar} \sum_{f} \frac{1}{\omega_{f n_{0}}^{2}-\omega^{2}} \sum_{l} \\
\left\{\frac{2 \operatorname{Re}\left[\left\{\left\langle n_{0} \vec{E}\left|d_{-}\right| f \vec{E}\right\rangle\left\langle f \vec{E}\left|d_{+}\right| l \vec{E}\right\rangle-\left\langle n_{0} \vec{E}\left|d_{+}\right| f \vec{E}\right\rangle\left\langle f \vec{E}\left|d_{-}\right| l \vec{E}\right\rangle\right\}\left\langle l \vec{E}\left|V_{w}\right| n_{0} \vec{E}\right\rangle\right]}{E_{n_{0}}-E_{l}}+\right. \\
\left.+\frac{2 \operatorname{Re}\left[\left\langle n_{0} \vec{E}\left|d_{-}\right| l \vec{E}\right\rangle\left\langle l \vec{E}\left|V_{w}\right| f \vec{E}\right\rangle\left\langle f \vec{E}\left|d_{+}\right| n_{0} \vec{E}\right\rangle-\left\langle n_{0} \vec{E}\left|d_{+}\right| l \vec{E}\right\rangle\left\langle l \vec{E}\left|V_{w}\right| f \vec{E}\right\rangle\left\langle f \vec{E}\left|d_{-}\right| n_{0} \vec{E}\right\rangle\right]}{E_{f}-E_{l}}\right\}
\end{gathered}
$$

It should be noted that the radial parts of the atom wave functions are real [9], therefore the matrix elements of the operators $d_{ \pm}$are real too.

As a result, we can conclude that the P-odd T-even part of the interaction $V_{w}$ does not give any contribution to $\beta_{E}^{T}$ because the P-odd T-even matrix elements for $V_{w}$ are imaginary [7]. At the same time, the T- and P-odd matrix elements for $V_{w}$ are real [7], therefore, the polarizability $\beta_{E}^{T} \neq 0$. It should be mentioned that in the absence of electric field $(\vec{E}=0)$ the polarizability $\beta_{E}^{T}=0$ and, therefore, the phenomenon of the photon polarization plane rotation is absent.

Really, an electric field $\vec{E}$ mixes atom levels with the opposite parity. The atom levels have the fixed parity when $\vec{E}=0$. The operators $d_{ \pm}$and $V_{w}$ change 
the parity of the atom states. As a result, the parity of a final state $\left|N_{0}^{\prime}\right\rangle=d_{+}$ $d_{-} V_{w}\left|N_{0}\right\rangle$ appears to be opposite to the parity of initial state $\left|N_{0}\right\rangle$. But the initial and final states in the expression for $\beta_{E}^{T}$ are the same. Therefore $\beta_{E}^{T}$ can not differ from zero when $\vec{E}=0$.

Let us estimate the magnitude of the T-odd photon plane rotation effect now. According to the analysis [4, 5, 6], based on the calculations of the value of $\mathrm{T}$ and $\mathrm{P}$ noninvariant interactions given by [7] the ratio $\frac{\left\langle V_{w}^{T}\right\rangle}{\left\langle V_{w}^{P}\right\rangle} \leq 10^{-3} \div 10^{-4}$, where $\left\langle V_{w}^{T}\right\rangle$ is $\mathrm{T}$ and P-odd matrix element, $\left\langle V_{w}^{P}\right\rangle$ is $\mathrm{P}$-odd T-even matrix element.

The P-odd T-even polarizability $\beta_{s}^{P}$ is proportional to the electric dipole matrix element, the magnetic dipole matrix element and $\left\langle V_{w}^{P}\right\rangle: \beta_{s}^{P} \sim\langle d\rangle\langle\mu\rangle\left\langle V_{w}^{P}\right\rangle$ [7. At the same time $\beta_{E}^{T} \sim\langle d(\vec{E})\rangle\langle d(\vec{E})\rangle\left\langle V_{w}^{T}\right\rangle$. As a result

$$
\frac{\beta_{E}^{T}}{\beta_{s}^{P}} \sim \frac{\langle d(\vec{E})\rangle\langle d(\vec{E})\rangle\left\langle V_{w}^{T}\right\rangle}{\langle d\rangle\langle\mu\rangle\left\langle V_{w}^{P}\right\rangle} .
$$

Let us study the T-odd phenomena of the photon polarization plane rotation in an electric field $\vec{E}$ for the transition $n_{0} \rightarrow f$ between the levels $n_{0}$ and $f$ which have the same parity when the field $\vec{E}=0$. In this case the matrix element $\left\langle n_{0}, \vec{E}\left|d_{ \pm}\right| f, \vec{E}\right\rangle$ does not equal to zero for $\vec{E} \neq 0$ only. Let the interaction energy of an atom with an electric field $V_{E}=-\vec{d} \vec{E}$ is much smaller then the spacing $\Delta$ of the energy levels, which are mixed by the field $\vec{E}$. It allows us to use the perturbation theory for the wave functions $|f, \vec{E}\rangle$ :

$$
|f, \vec{E}\rangle=|f\rangle+\sum_{m} \frac{\left\langle m\left|-d_{z} E\right| f\right\rangle}{E_{f}-E_{m}}|m\rangle,
$$

where $z$ axis is parallel to $\vec{E}$.

As a result, the matrix element $\left\langle n_{0}, \vec{E}\left|d_{ \pm}\right| f, \vec{E}\right\rangle$ can be written as:

$$
\begin{aligned}
\left\langle n_{0}, \vec{E}\left|d_{ \pm}\right| f, \vec{E}\right\rangle= & \\
= & -\left\{\sum_{m} \frac{\left\langle n_{0}\left|d_{ \pm}\right| m\right\rangle\left\langle m\left|d_{z}\right| f\right\rangle}{E_{f}-E_{m}}+\right. \\
& \left.+\sum_{p} \frac{\left\langle n_{0}\left|d_{z}\right| p\right\rangle\left\langle p\left|d_{ \pm}\right| f\right\rangle}{E_{n_{0}}-E_{p}}\right\} E .
\end{aligned}
$$

One can see that the matrix element $\langle d(\vec{E})\rangle \sim \frac{\langle d\rangle E}{\Delta}\langle d\rangle$ in this case.

The others matrix elements in (16) we can calculate for $\vec{E}=0$. 
As a result, we can obtain the estimation

$$
\beta_{E}^{T} \sim\langle d\rangle\langle d\rangle \frac{\langle d E\rangle}{\Delta}\left\langle V_{w}^{T}\right\rangle
$$

The ratio (17) can be written as

$$
\frac{\beta_{E}^{T}}{\beta_{s}^{P}} \sim \frac{\langle d\rangle\langle d\rangle \frac{\langle d E\rangle}{\Delta}\left\langle V_{w}^{T}\right\rangle}{\langle d\rangle\langle\mu\rangle\left\langle V_{w}^{P}\right\rangle}
$$

The matrix element $\langle\mu\rangle \sim \alpha\langle d\rangle$ [8, 9], where $\alpha=\frac{1}{137}$ is the fine structure constant. Finally we have:

$$
\frac{\beta_{E}^{T}}{\beta_{s}^{P}} \sim \alpha^{-1} \frac{\langle d E\rangle}{\Delta} \frac{\left\langle V_{w}^{T}\right\rangle}{\left\langle V_{w}^{P}\right\rangle}
$$

For the case $\frac{\langle d E\rangle}{\Delta} \sim 1$ the ratio $(22)$ gives

$$
\frac{\beta_{E}^{T}}{\beta_{s}^{P}} \sim \alpha^{-1} \frac{\left\langle V_{w}^{T}\right\rangle}{\left\langle V_{w}^{P}\right\rangle} \lesssim 10^{-1} \div 10^{-2}
$$

This is possible, for example, for exited states of atoms and for two-atom molecules (TlF, BiS, $\mathrm{HgF}$ ). As one can see, the ratio $\frac{\beta_{E}^{T}}{\beta_{s}^{P}}$ is two orders larger as compared with the simple estimation $\frac{\left\langle V_{w}^{T}\right\rangle}{\left\langle V_{w}^{P}\right\rangle} \leq 10^{-3} \div 10^{-4}$ due to the fact that $\beta_{E}^{T}$ is determined by the electric dipole transitions only, while $\beta_{s}^{P}$ is determined both by the electric and magnetic dipole transitions. As a result, the experimental observation of the T-odd photon polarization plane rotation becomes real. The effect can be increased by using a resonator or a volume diffraction grating $[6]$.

The absorption of the photon in the matter does not allow to do the length $L$ significantly greater then the absorption length $L_{a}$. This difficulty can be removed when the photon passes in the medium with the inverse population of the atom states (the conditions of laser generation or amplification are fulfilled for the transitions of our interest). The coherent wave is not absorbed in this case, it is amplified. As a result the length $L$ can be done significantly greater then $L_{a}$. We can use the high quality resonators and diffraction gratings for the effective enhancement of the length $L$. Let us note that due to T-odd interactions the amplification depends on the photon circular polarization sign. So we can study two effects in laser medium: T-odd photon plane rotation in the electric field $\vec{E}$ and circular dichroism - different amplification for the left and right photons. The atoms $\mathrm{Cs}, \mathrm{Tl}, \mathrm{Pb}, \mathrm{Dy}$ are very convenient for this as well as for P-odd T-even phenomena studying [4, 6, 6]. It is important, that for such atoms laser generation has been done. 
5. Thus we have shown that the T-odd and P-odd phenomena of photons polarization plane rotation and circular dichroism are not very small in comparison with P-odd T-even effects. As a result, we can hope to measure them experimentally. It is convenient to use atoms $\mathrm{Cs}, \mathrm{Tl}, \mathrm{Pb}, \mathrm{Dy}$ and two-atom molecules containing heavy atom $\mathrm{TlF}, \mathrm{BiS}, \mathrm{HgF}$, DyF for the investigation of $\mathrm{P}, \mathrm{T}$-odd phenomena as well as for the investigation of P-odd T-even ones [7].

Let us note, that the new T-odd and P-odd phenomena of photon polarization plane rotation (circular dichroism) in an electric field has general meaning. So, the super strong electric fields are formed in the collisions of the relativistic nuclei. The $\gamma$-quanta originating in such collisions propagate in the nuclear matter which interacts with the electric field. As a result the radiated $\gamma$-quanta have the circular polarization. The gravitational field stipulates the appearance of the gravitational analog of the Stark mixing of atom (molecule) levels. It means that the gravitational analog of the T-odd polarizability $\beta_{g}^{T}$ exists. As a result, the T-odd P-odd phenomena of photon plane rotation (circular dichroism) appears, for example, for an electromagnetic wave moving in a matter interacting with the strong gravitational field of a star (term in the scattering amplitude proportional to $\beta_{g}^{T}(\vec{g}[\vec{e} * \times \vec{e}]), \vec{g}$ is the free fall acceleration). Due to quantum electrodynamic effects of electron-positron pair creation in strong electric and magnetic fields the dielectric permittivity tensor $\varepsilon_{i k}$ of vacuum depends on $\vec{E}$ and $\vec{H}$ [8]. The theory of $\varepsilon_{i k}$ [8] does not take into account the weak interaction of electron and positron with each other. Considering the weak interactions of electron and positron with each other in the process of pair creation in an electric field one can obtain, that the permittivity tensor of vacuum in strong electric (gravitational) field contains the term $\beta_{v a c}^{T} \vec{E}\left(\overrightarrow{n_{E}}[\vec{e} * \times \vec{e}]\right)$ , $\varepsilon_{i k}^{v a c} \sim i \beta_{v a c \vec{E}}^{T} \varepsilon_{i k l} n_{l E}\left(\beta_{v a c \vec{g}}^{T}\left(\vec{g}\left[\vec{e}^{*} \times \vec{e}\right]\right), \varepsilon_{i k}^{v a c} \sim i \beta_{v a c}^{T} \vec{g} \varepsilon_{i k l} \vec{g}\right)$, and as a result, the polarization plane rotation (circular dichroism) phenomena exist for photon moving in an electric (gravitational) field in vacuum. And visa versa $\gamma$-quanta appeared while single-photon electron-positron annihilation in an electric (gravitational) field will have the admixture of circular polarization, caused by T-odd P-odd weak interactions.

\section{References}

[1] 1. Christenson J.H., Cronin J.W., Fitch V.L. and Turlay R. Phys. Rev. Lett. / 3 (1964) 1138.

[2] Lamoreaux S.K. Nucl. Instrum. Methods. A284 (1989) 43.

[3] Forte M.J. Journ. Of Phys. G.: Nucl. Part. Phys. G9 (1983) 745.

[4] Baryshevsky V.G. Phys. Lett A177 (1993) 38.

[5] Baryshevsky V.G., Baryshevsky D.V. Journ. Of Phys. B: At. Mol. Opt. Phys. 27 (1994) 4421.

[6] Baryshevsky V.G. J.High Energy Phys. 04 (1998) 018. 
[7] Khriplovich I.B. Parity Nonconservation in Atomic Phenomena. 1991 (London: Gordon and Breach).

[8] Berestetskii V., Lifshitz E., Pitaevskii L. Quantum electrodynamics, 1989, Moscow Science.

[9] Landau L., Lifshitz E. Quantum mechanics, 1989, Moscow Science. 